\title{
Association Between Central Sensitization and Increasing Prevalence of Nocturnal Knee Pain in the General Population with Osteoarthritis from the Iwaki Cohort Study
}

\author{
Eiji Sasaki (1D' \\ Seiya Ota ${ }^{1,2}$ \\ Daisuke Chiba' \\ Yuka Kimura (iD) \\ Shizuka Sasaki ${ }^{1}$ \\ Masataka Ando ${ }^{2}$ \\ Yuji Yamamoto' \\ Eiichi Tsuda ${ }^{3}$ \\ Yasuyuki Ishibashi ${ }^{1}$ \\ 'Department of Orthopaedic Surgery, \\ Hirosaki University Graduate School of \\ Medicine, Hirosaki, Japan; ${ }^{2}$ Department \\ of Social Medicine, Hirosaki University \\ Graduate School of Medicine, Hirosaki, \\ Japan; ${ }^{3}$ Department of Rehabilitation \\ Medicine, Hirosaki University Graduate \\ School of Medicine, Hirosaki, Japan
}

\begin{abstract}
Purpose: Knee pain is associated with osteoarthritis (OA) and increases during this condition; however, its correlation with central sensitization (CS) in arthritis patients requires greater understanding. The present cross-sectional cohort study to explore the prevalence of knee OA, nocturnal knee pain and disability in general population and to examine the association of CS with sleep quality in Japanese general population.

Patients and Methods: From among 1056 community-dowelling volunteers, 942 were enrolled as participants in this study. Bilateral weight-bearing anterior-posterior knee radiographs were classified by the Kellgren-Lawrence grade. Nocturnal knee pain and disability were assessed with self-reported questionnaires. Using the CS inventory with nine items (CSI-9), CS was defined as 10 points or higher. Sleep quality was scored using the Pittsburgh Sleep Quality Index (PSQI). Linear regression analysis, adjusted by age, sex, body mass index, Kellgren-Lawrence grade, nocturnal knee pain, and lifestyle habits, was performed to investigate the association of CS with PSQI.

Results: The prevalence of OA, nocturnal knee pain, and disability was $37.9 \%, 7.6 \%$, and $6.2 \%$, respectively. The mean CSI-9 score was $4.9 \pm 4.4$, with a CS prevalence of $14.0 \%$. The mean PSQI score was $3.9 \pm 2.4$, which was correlated with the CSI-9 value. CS was not correlated with OA severity; however, nocturnal knee pain prevalence increased from $13.3 \%$ to $25.5 \%$ in knee OA patients with CS. The CSI-9 value correlated with PSQI total score and subscales.

Conclusion: Knee OA severity correlated with nocturnal pain and disability; however, its association with CS was unproven. The combined effect of knee OA and CS elevated nocturnal pain and disability, resulting in diminished sleep quality.
\end{abstract}

Keywords: central sensitization, epidemiology, knee osteoarthritis, nocturnal knee pain, sleep quality

\section{Introduction}

Knee osteoarthritis (OA) typically causes chronic pain and disability in the elderly. ${ }^{1}$ The radiographic prevalence of knee OA among adults aged $>40$ years has been reported to be approximately $42.0 \%$ in men and $62.4 \%$ in women. ${ }^{2}$ The progression of knee OA leads to irreversible structural changes, high treatment costs for joint replacement, reduced productivity, and absence from work; therefore, it contributes to a growing substantial burden on the society. ${ }^{3}$ Knee pain with OA is related to multiple confounders, ${ }^{4}$ and it is known that a weak association exists between the
Correspondence: Eiji Sasaki

Department of Orthopaedic Surgery, Hirosaki University Graduate School of Medicine, 5 Zaifu-cho, Hirosaki, Aomori, 036-8562, Japan

Tel +8I- I72-39-5083

Fax +8I-I72-36-3826

Email e_sasaki@hirosaki-u.ac.jp 
degree of joint damage and the presence and intensity of clinical pain. ${ }^{5,6}$ This constitutes one of the reliable predictors of knee OA-associated disability. ${ }^{7-9}$ Nocturnal pain and disability are typical symptoms of knee OA; which diminish satisfaction over the knee condition and quality of life (QOL). ${ }^{10-13}$ As chronic pain or nocturnal knee symptoms have considerable effects on the QOL, appropriate understanding and evaluation of chronic pain are critical for its management and control. The etiology and epidemiological background need to be evaluated further.

Recently, central sensitization (CS) has received attention as one of the key factors for recognizing chronic pain in knee OA patients. ${ }^{14,15}$ Previous studies have revealed that CS induces hyperalgesia and widespread pain in OA patients. $^{14,15}$ According to the International Association for the Study of Pain, CS is defined as the enhanced responsiveness of nociceptors in the central nervous system to typical, non-noxious, or subthreshold afferent stimulation input. ${ }^{16}$ This results in hypersensitivity and increased pain response outside the area of injury, ie, an expanded receptive field. ${ }^{17,18}$ The intrinsic risk of CS in OA patients at the terminal stage was estimated to be approximately $20 \%{ }^{19}$ this potentially led to subsequent impairments in the QOL. ${ }^{20}$ Indeed, certain OA patients present a psychological background, such as catastrophizing or depressive conditions, for chronic pain and nocturnal knee symptoms. ${ }^{21} \mathrm{CS}$ may be related to nocturnal pain; ${ }^{22}$ nonetheless, the association of CS with such symptoms in knee OA is yet to be evinced from a large-sample epidemiological study. In addition, the correlation of CS with radiographic knee OA severity remains unclear.

Therefore, this cross-sectional epidemiological study aimed to explore the prevalence of knee OA, nocturnal knee pain and disability in the general population and to examine the association of CS with sleep quality. The epidemiological nature of the study made evaluating these associations possible because a large general population with or without any stage of OA and symptoms could be included. We hypothesized that the prevalence of CS increases with radiographic knee OA severity, similar to that of nocturnal symptoms, with a concomitant decrease in sleep quality.

\section{Materials and Methods}

\section{Participants}

Held since 2008, the Iwaki Health Promotion Project is an annual community-based preventive medicine program that aims to improve average life expectancy., ${ }^{9,23}$ of approximately 10,000 people in the general population living in the Iwaki area of Hirosaki, located in western Aomori Prefecture, Japan, 1056 volunteers participating in the general health check-ups of the Iwaki Health Promotion Project from June 26 to July 4, 2018 were recruited through mass media advertisements and by public health nurses, and were screened for inclusion in this study. Individuals were excluded if they were postoperative for total knee arthroplasty or arthrodesis; received treatment for rheumatoid arthritis or psychological disorders; had malignant tumors; or had incomplete or unavailable data. For anthropometric evaluation, height and weight were measured and recorded to calculate the body mass index (BMI). In addition, the summed skeletal muscle volume of the trunk, lower extremity, and upper extremity was measured using a body composition analyzer (Tanita MC-190; Tanita Corp., Tokyo, Japan). Skeletal muscle index (SMI) was calculated as follows: SMI $\left(\mathrm{kg} / \mathrm{m}^{2}\right)=$ skeletal muscle mass $(\mathrm{kg}) /$ height $^{2}$ (meters). This study was performed in accordance with the 1964 Helsinki Declaration and its later amendments or comparable ethical standards. Ethical approval was obtained from the ethics committee of the Hirosaki University Graduate School of Medicine. Written informed consent was obtained from all participants.

\section{Radiographic Evaluation}

Radiographic examination of both knees was performed using the CXDI-40EG digital radiography system (Canon Inc., Tokyo, Japan). ${ }^{23}$ Experienced radiologic technicians and orthopedic surgeons obtained weight-bearing anterior-posterior radiographs at maximum extension with foot map positioning. The beam was placed parallel to the floor and aimed at the joint space; the sequencing was set at $60 \mathrm{kV}, 50 \mathrm{~mA}$, and $80 \mathrm{~ms}$. Images obtained were converted into the joint photographic experts group (.jpeg) file format. OA severity was evaluated as KellgrenLawrence (KL) grades (from 0 to 4 ) using the KL radiographic atlas; this was performed by two trained orthopedic surgeons (D.C. and S.O.). ${ }^{24}$ Participants rated with a $\mathrm{KL}$ grade of 2 and above were categorized into the OA group, while those rated with a KL grade of 0 and 1 were categorized into the non-OA group. Furthermore, based on the radiographic knee OA severity of their worse knee, participants were classified into the normal $(\mathrm{KL}$ grade $=0$ or 1$)$, moderate $(\mathrm{KL}$ grade $=2)$, and severe (KL grade $=3$ or 4 ) groups. $^{25}$ Regarding the interclass correlation coefficient (ICC) between the two surgeons, the ICC $(2,1)$ was 0.815 . Surgeons were blinded to the 
sequence of radiograph acquisition and the clinical status of the participants.

\section{Central Sensitization}

CS was evaluated using the central sensitization inventory A (CSI) with self-reported questionnaires. ${ }^{26}$ CSI displays satisfactory psychometric strength, clinical utility, and initial construct validity. The completed version comprises 25 items; however, the shorter version was locally available and consists of nine items (CSI-9). Scores were assigned from 0 to 4 , and calculated to 100 points in CSI full version and 36 points in CSI-9, with a higher score indicating a more severe CS condition. The CSI-9 was validated using the Spearman correlation coefficient $(\mathrm{r}=$ 0.91) using the full version of CSI. ${ }^{27}$ Based on this previous report, ${ }^{27}$ we categorized patients into the CS group if they had a score of 10 points or higher.

\section{Knee Osteoarthritis Symptoms and Nocturnal Pain}

Knee OA symptoms were evaluated using a patient-based tool (Knee Injury and Osteoarthritis Outcome Score [KOOS]) and considered under five subscales (pain, symptoms, activities of daily living, sports/recreation, and QOL). ${ }^{28,29}$ Nocturnal knee pain was evaluated using the question: "At night, while in bed, how much knee pain did you experience during the last week?" Similarly, nocturnal disability during movement as part of activities of daily living was assessed using the question: "Last week, while lying in bed, how much difficulty did you experience while turning over or maintaining the knee position?" In these questionnaires, the participants selected the most suitable option among "none", "mild", "moderate", "severe", and "extreme"; if they chose the mild, moderate, severe, and extreme options, they were defined to have nocturnal knee pain or disability. The prevalence of such symptoms was calculated for all participants and for those with knee OA.

\section{Sleep Quality}

Sleep quality was evaluated using the Pittsburgh Sleep Quality Index (PSQI), which is a valid and reliable index for patients with arthritis. ${ }^{30}$ The PSQI consists of seven subscales for sleep evaluation: quality, latency, duration, habitual efficiency, disturbance, use of medication, and daytime dysfunction. ${ }^{31,32}$

\section{Statistical Analysis}

Demographic data of the non-CS and CS groups were expressed as means \pm standard deviations. Comparisons were performed using the chi-square test for categorical variables and the Mann-Whitney $U$-test for continuous variables. The prevalence of CS was calculated among the following age-specific groups (years): 18-39 (106 men and 141 women), 40-49 (81 men and 89 women), 50-59 (66 men and 109 women), 60-69 (90 men and 131 women), and $\geq 70$ (51 men and 75 women). Spearman correlation coefficients ( $r$ ) were estimated between agespecific groups and prevalence of CS. Additionally, nocturnal knee pain and disability among the three categories of OA (KL grades $0 / 1,2$, and 3/4) were compared using the chi-square test. The chi-square test was also performed to investigate the prevalence of nocturnal knee pain and disability in those with or without knee OA or CS, and the influence of CS on the prevalence of nocturnal knee symptoms, between non-CS and CS groups in those with knee OA. The Spearman correlation coefficients between the PSQI and the CSI-9 scores were estimated. To investigate the factors related to nocturnal symptoms in the non-OA and OA groups, crude and adjusted logistic regression analyses were performed. The adjusted logistic regression model included age, sex, BMI, KL grade, CS, and lifestyle habits as covariates. Furthermore, to reveal the association of CS with sleep quality, linear regression analysis was performed with the seven subscales and aggregate score of the PSQI as the dependent variables and the CSI-9 value as the independent variable in all participants and in those with OA. Each subscale was adjusted for age, sex, BMI, KL grade, nocturnal knee pain, and lifestyle habits. Ultimately, to estimate the cut-off PSQI score for the determination of the presence of CS, receiver operating characteristic (ROC) analysis was performed with the total PSQI score as the testing variable and the presence of CS as the diseased condition. The area under the curve (AUC) was calculated; the cut-off value was defined as Youden's index based on the sensitivity and specificity. Data input and analysis were performed using SPSS version 25.0 J (SPSS Inc., Chicago, IL, USA). A p-value (p) $<0.05$ was considered statistically significant.

\section{Results}

Of the 1056 participants screened, 114 were excluded because they received rheumatoid arthritis treatment (13), underwent postoperative total knee arthroplasty and 
arthrodesis (9), were treated for psychological disorders (10), had malignant tumors (36), and had incomplete or unavailable data (46). Consequently, 942 participants (394 men and 548 women) were included, and their data were collected for statistical analysis. Knee OA, nocturnal knee pain, and disability were observed in 357 (37.9\%), 72 $(7.6 \%)$, and $58(6.2 \%)$ participants, respectively. Furthermore, the overall mean CSI-9 score was $4.9 \pm 4.4$ points (range: $0-31$ points) and the overall CS prevalence was $14.0 \%$. With aging, the prevalence of CS increased in men $(r=0.894, p=0.041)$ but decreased in women $(\mathrm{r}=-0.872, \mathrm{p}=0.054)$ (Figure 1). The mean PSQI score was $3.9 \pm 2.4$ points (range: $0-18$ points) and was positively correlated with the CSI-9 score $(r=0.390, p<$ 0.001). Participants with a positive CSI-9 score had a lower SMI with depleted KOOS subscales $(p=0.015$ and $\mathrm{p}<0.001$, respectively) and a higher PSQI score ( $\mathrm{p}<$ 0.001) (Table 1).

With respect to radiographic severity, $62.1 \%, 32.2 \%$, and $5.7 \%$ of the participants had KL grades of $0 / 1,2$, and $3 / 4$, respectively. The prevalence of nocturnal pain $(\mathrm{p}<$ $0.001)$ and disability $(\mathrm{p}<0.001)$ increased with knee OA severity; however, similar changes did not occur with CS $(\mathrm{p}=0.537)($ Table 2).

With knee OA, the prevalence of nocturnal pain and disability increased up to $25.5 \%$ (9.1 times, $\mathrm{p}=0.021)$ and $21.8 \%$ (18.2 times, $p=0.033$ ), respectively (Figure 2 ). The adjusted logistic regression analysis revealed that CS was significantly correlated with nocturnal knee pain in the OA group ( $\mathrm{p}=0.008$, odds ratio: $2.73,95 \%$ confidence interval [CI]: 1.30-5.72) (Tables 3 and 4). Furthermore, CS was significantly correlated with nocturnal disability in the OA group

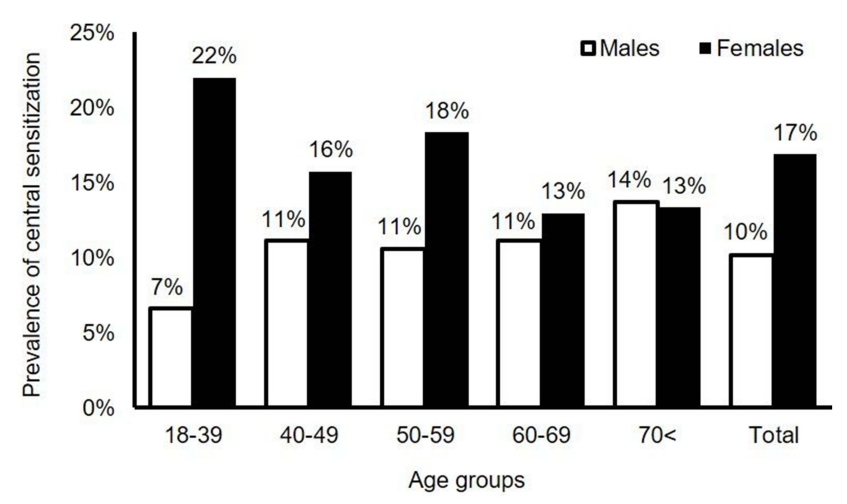

Figure I Prevalence of central sensitization (CS) according to age-specific groups. This was defined using CS inventory 9 (CSI-9). A score of 10 points or higher was regarded as positive.
Table I Demographic Data of the Study Participants

\begin{tabular}{|l|c|c|c|}
\hline & Non-CS & CS & p-value \\
\hline Sample Number & $\mathbf{8 1 0}$ & 132 & \\
\hline Age (y.o.) & $52.27 \pm 15.5$ & $51.1 \pm 15.3$ & 0.462 \\
Females (\%) & $456(56.3 \%)$ & $92(69.7 \%)$ & 0.004 \\
Fat (\%) & $25.5 \pm 8.0$ & $26.7 \pm 8.5$ & 0.119 \\
Skeletal muscle index (kg/m2) & $16.0 \pm 2.1$ & $15.5 \pm 1.8$ & 0.015 \\
Body mass index (kg/m2) & $22.9 \pm 3.5$ & $22.7 \pm 3.8$ & 0.375 \\
KOOS Symptom & $91.9 \pm 12.6$ & $87.6 \pm 13.9$ & $<0.001$ \\
KOOS Pain & $93.6 \pm 12.7$ & $89.4 \pm 15.2$ & $<0.001$ \\
KOOS ADL & $96.8 \pm 8.7$ & $92.7 \pm 13.1$ & $<0.001$ \\
KOOS Sports & $91.7 \pm 18.5$ & $84.4 \pm 22.4$ & $<0.001$ \\
KOOS QOL & $86.6 \pm 19.9$ & $78.2 \pm 22.7$ & $<0.001$ \\
PSQI & $3.6 \pm 2.1$ & $6.0 \pm 2.9$ & $<0.001$ \\
Central sensitization inventory full & $7.4 \pm 5.9$ & $26.0 \pm 8.3$ & $<0.001$ \\
Central sensitization inventory 9 & $3.6 \pm 2.8$ & $13.0 \pm 3.3$ & $<0.001$ \\
Smoking habit (\%) & $136(16.8 \%)$ & $24(18.2 \%)$ & 0.708 \\
Drinking habit (\%) & $383(47.3 \%)$ & $52(39.4 \%)$ & 0.109 \\
Fitness habit (\%) & $143(17.7 \%)$ & $20(15.2 \%)$ & 0.536 \\
\hline
\end{tabular}

Notes: Continuous variables are expressed as means \pm standard deviations and compared using the Mann-Whitney U-test. Categorical variables were compared between the non-CS and CS groups using the Chi-square test.

( $p=0.009$, odds ratio: $2.94,95 \%$ CI: $1.31-6.57)$ and non-OA $(\mathrm{p}=0.001$, odds ratio: $11.24,95 \% \mathrm{CI}: 2.84-44.73)$ groups.

In the OA group, the CSI-9 score was associated with the aggregate PSQI score $(p \leq 0.001)$ and with the subscales of sleep quality ( $p \leq 0.001)$, sleep latency $(p \leq 0.001)$, sleep disturbance $(\mathrm{p}=0.003)$, use of sleeping medication $(\mathrm{p}=0.002)$, and daytime dysfunction $(\mathrm{p} \leq 0.001)$ (Table 5). The ROC analysis showed that PSQI moderately reflected the presence of CS ( $p \leq 0.001$, AUC: $0.750,95 \%$ CI: $0.713-$ 0.801 ), and indicated a PSQI score of 4 as the cut-off value based on a sensitivity of 0.833 and specificity of 0.553 (Odds ratio: 4.43) (Figure 3).

\section{Discussion}

The most significant finding of the present study was that the prevalence of CS did not increase with increasing

Table 2 Prevalence of Nocturnal Symptoms and Central Sensitization (CS) in Terms of Radiographic Knee Osteoarthritis (OA) Severity

\begin{tabular}{|l|c|c|c|c|}
\hline & KL 0/I & KL 2 & KL3/4 & p-value \\
\hline Sample & $\mathbf{5 8 5}$ & $\mathbf{3 0 3}$ & $\mathbf{5 4}$ & \\
\hline Central sensitization & $77(13.2 \%)$ & $48(15.8 \%)$ & $7(13.2 \%)$ & 0.537 \\
Nocturnal knee pain & $18(3.1 \%)$ & $37(12.2 \%)$ & $17(31.5 \%)$ & $<0.001$ \\
Nocturnal disability & $12(2.1 \%)$ & $30(9.9 \%)$ & $16(29.6 \%)$ & $<0.001$ \\
\hline
\end{tabular}

Notes: The prevalence of CS, nocturnal knee pain, and disability with respect to knee OA severity were compared using the chi-square test. 
A

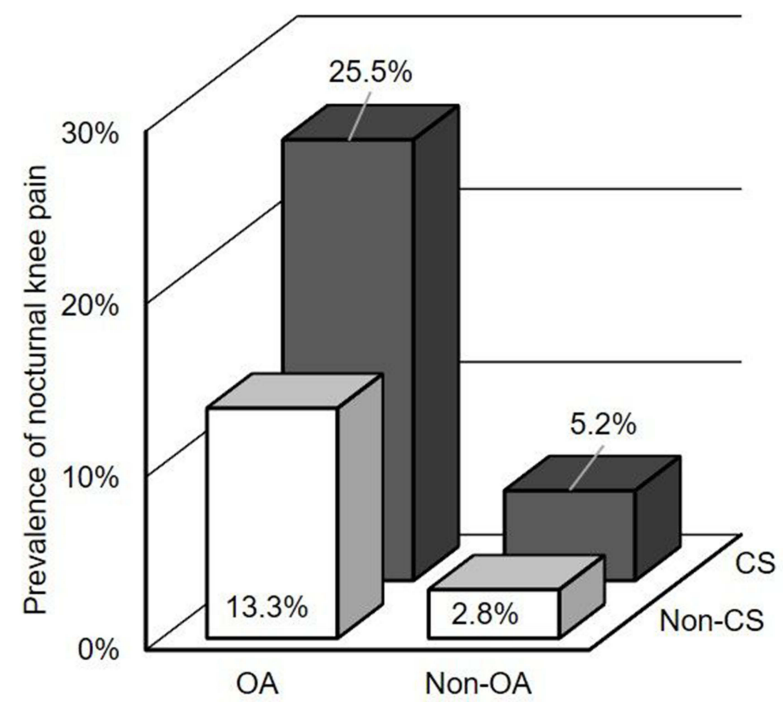

B

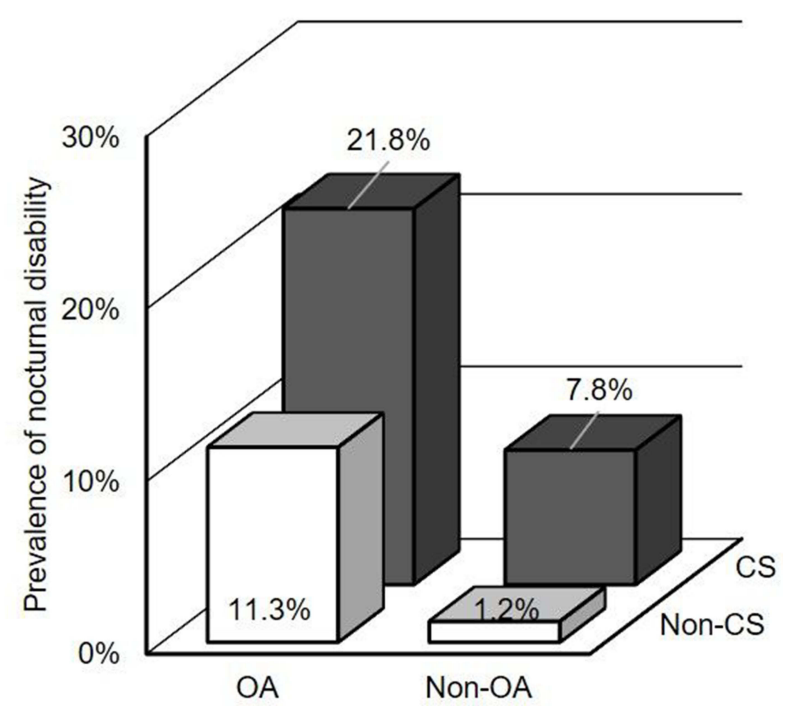

Figure 2 Prevalence of nocturnal knee pain (A) and disability (B) in the central sensitization (CS) and knee osteoarthritis (OA) groups. Abbreviations: CS, central sensitization; OA, osteoarthritis.

radiographic knee OA severity; however, the presence of CS was correlated with an additional high prevalence of nocturnal knee symptoms in individuals with knee OA. Furthermore, the presence of CS was also associated with lower sleep quality. ROC analysis revealed that a PSQI score of 4 points or higher was a significant risk factor for potential CS. Irrespective of the radiographic knee OA severity, orthopedic surgeons should carefully evaluate the potential presence of CS, as it is related to postoperative poor outcomes. ${ }^{19,33,34}$
Nocturnal symptoms such as pain due to knee OA immensely impact the QOL. ${ }^{10}$ This was the ninth-most frequently reported complaint in knee OA patients; the pain itself was infrequent and did not bother these patients. ${ }^{11}$ In another study, the majority of hip and knee OA patients reported experiencing nocturnal pain through an interview-style investigation. ${ }^{12}$ A large-sample cohort study featuring 118,336 subjects with arthritis reported that $17 \%$ of adults $\geq 18$ years of age were usually experiencing some level of knee pain, which was related to

Table 3 Factors Related to the Presence of Nocturnal Knee Pain

\begin{tabular}{|l|c|c|c|c|c|c|c|c|}
\hline \multirow{2}{*}{} & \multicolumn{4}{|c|}{ Non-OA } & \multicolumn{4}{c|}{ OA } \\
\cline { 2 - 8 } & B & p-value & Odds & $95 \%$ Cl & B & p-value & Odds & $95 \%$ CI \\
\hline Crude & & & & & & & \\
\hline Adjusted & 0.66 & 0.256 & 1.93 & $0.62-6.03$ & 0.81 & $<0.001$ & 2.24 & $1.12-4.47$ \\
Age & & & & & & & \\
Females & 0.03 & 0.103 & 1.03 & $0.99-1.07$ & 0.04 & 0.005 & 1.04 & $1.01-1.07$ \\
BMI & -0.96 & 0.142 & 0.38 & $0.11-1.38$ & 0.10 & 0.799 & 1.10 & $0.53-2.30$ \\
KL grade & 0.01 & 0.862 & 1.01 & $0.86-1.19$ & 0.04 & 0.377 & 1.04 & $0.95-1.13$ \\
Central sensitization & 0.51 & 0.376 & 1.67 & $0.54-5.19$ & 0.52 & 0.129 & 1.68 & $0.86-3.28$ \\
Smoking habit & 0.96 & 0.118 & 2.62 & $0.78-8.75$ & 1.00 & 0.008 & 2.73 & $1.30-5.72$ \\
Drinking habit & -0.08 & 0.906 & 0.92 & $0.24-3.60$ & 0.33 & 0.562 & 1.38 & $0.46-4.15$ \\
Fitness habit & -0.96 & 0.099 & 0.38 & $0.12-1.20$ & 0.14 & 0.703 & 1.14 & $0.57-2.29$ \\
\hline
\end{tabular}

Notes: Crude and adjusted logistic regression analyses were performed with nocturnal knee pain as the dependent variable and age, sex, body mass index (BMI), KellgrenLawrence (KL) grade, central sensitization (CS), and lifestyle habits as the independent variables in the non-OA $(n=585)$ and OA $(n=357)$ groups. Abbreviation: $95 \% \mathrm{Cl}, 95 \%$ confidence interval. 
Table 4 Factors Related to the Presence of Nocturnal Disability

\begin{tabular}{|c|c|c|c|c|c|c|c|c|}
\hline & \multicolumn{4}{|c|}{ Non-OA } & \multicolumn{4}{|c|}{ OA } \\
\hline & B & p-value & Odds & $95 \% \mathrm{Cl}$ & B & p-value & Odds & $95 \% \mathrm{Cl}$ \\
\hline \multicolumn{9}{|l|}{ Crude } \\
\hline Central sensitization & 1.96 & $<0.001$ & 7.07 & $2.22-22.52$ & 0.79 & 0.035 & 2.20 & $1.06-4.58$ \\
\hline \multicolumn{9}{|l|}{ Adjusted } \\
\hline Age & 0.09 & 0.004 & 1.09 & $1.03-1.16$ & 0.06 & $<0.001$ & 1.06 & $1.03-1.09$ \\
\hline Females & -0.16 & 0.869 & 0.86 & $0.14-5.41$ & 0.09 & 0.830 & 1.09 & $0.48-2.47$ \\
\hline $\mathrm{BMI}$ & 0.05 & 0.727 & 1.05 & $0.8 I-1.36$ & 0.05 & 0.308 & 1.05 & $0.96-1.16$ \\
\hline $\mathrm{KL}$ grade & -0.70 & 0.351 & 0.50 & $0.12-2.16$ & 0.48 & 0.184 & 1.61 & $0.80-3.25$ \\
\hline Central sensitization & 2.42 & 0.001 & 11.24 & $2.84-44.73$ & 1.08 & 0.009 & 2.94 & $1.31-6.57$ \\
\hline Smoking habit & -0.06 & 0.971 & 0.00 & $0.14-6.34$ & 0.52 & 0.622 & 0.70 & $0.50-5.7$ I \\
\hline Drinking habit & -1.01 & 0.858 & 1.39 & $0.07-1.96$ & -0.19 & 0.401 & 0.22 & $0.38-1.82$ \\
\hline Fitness habit & 0.18 & 0.837 & 1.20 & $0.21-6.8 I$ & -0.85 & 0.097 & 0.43 & $0.16-1.17$ \\
\hline
\end{tabular}

Notes: Crude and adjusted logistic regression analyses were performed with nocturnal disability as the dependent variable and age, sex, body mass index (BMI), KellgrenLawrence $(K L)$ grade, central sensitization $(C S)$, and lifestyle habits as the independent variables in the non-OA $(n=585)$ and OA $(n=357)$ groups.

Abbreviation: $95 \% \mathrm{Cl}, 95 \%$ confidence interval.

Table 5 Influence of Central Sensitization (CS) on Sleep Quality

\begin{tabular}{|c|c|c|c|c|c|c|}
\hline \multirow[t]{2}{*}{ Dependent Variables } & \multicolumn{3}{|c|}{ Overall } & \multicolumn{3}{|c|}{ OA } \\
\hline & $\beta$ & p-value & Adjusted $\mathbf{R}^{2}$ & $\beta$ & p-value & Adjusted $\mathbf{R}^{2}$ \\
\hline Sleep quality & 0.36 & $<0.001$ & 0.14 & 0.23 & $<0.001$ & 0.11 \\
\hline Sleep latency & 0.24 & $<0.001$ & 0.06 & 0.20 & $<0.001$ & 0.05 \\
\hline Sleep duration & 0.13 & $<0.001$ & 0.05 & 0.07 & 0.174 & 0.05 \\
\hline Habitual sleep efficiency & 0.01 & 0.85 & 0.01 & 0.05 & 0.381 & -0.01 \\
\hline Sleep disturbance & 0.28 & $<0.001$ & 0.1 & 0.16 & 0.003 & 0.05 \\
\hline Use of sleeping medication & 0.22 & $<0.001$ & 0.06 & 0.16 & 0.002 & 0.04 \\
\hline Daytime dysfunction & 0.38 & $<0.001$ & 0.15 & 0.34 & $<0.001$ & 0.12 \\
\hline PSQI total & 0.41 & $<0.001$ & 0.19 & 0.32 & $<0.001$ & 0.11 \\
\hline
\end{tabular}

Notes: Linear regression analysis was performed with the seven subscales and the aggregate Pittsburgh Sleep Quality Index (PSQI) score as the dependent variables and with the CSI-9 score as the independent variable in all participants $(n=942)$ and in those with OA $(n=357)$. Each subscale was adjusted for age, sex, body mass index (BMI), Kellgren-Lawrence $(\mathrm{KL})$ grade, nocturnal knee pain, drinking, smoking, and fitness habits. $\beta$ indicates the standardized partial regression coefficients.

insomnia symptoms. ${ }^{13}$ In addition, a previous study showed that the prevalence of nocturnal pain increased with radiographic knee OA severity. ${ }^{10}$ In the present study, the combination of $\mathrm{CS}$ with radiographic knee OA was shown to increase the prevalence of nocturnal knee pain and disability in comparison with OA patients without CS. This result suggests that the CS on radiographic knee OA additionally increases the prevalence of nocturnal knee pain, while OA severity correlates to an increased prevalence of nocturnal knee pain. Furthermore, this study revealed that the combination of $\mathrm{OA}$ and $\mathrm{CS}$ diminished sleep quality. Only one-third of the participants with radiographic knee OA have previously reported symptoms; ${ }^{6}$ their severity was considered to be influenced by $\mathrm{CS}$ and radiographic knee $\mathrm{OA}$. In the clinical settings, persistent intense nocturnal knee pain is regarded as an indication for total arthroplasty ${ }^{35,36}$ for providing relief. ${ }^{37}$ In addition, reports that duloxetine administration to hip and knee OA patients reduced nocturnal pain indirectly support our results. ${ }^{38,39}$ These findings suggest that the presence of CS is consistently associated with increasing nocturnal knee symptoms, regardless of radiographic severity, resulting in a diminished sleep quality.

In patients with nocturnal knee symptoms, the overriding concern with $\mathrm{CS}$ is pain sensitization, which induces signs of localized and widespread stimulation. 5,40 However, the association of radiographic knee OA severity with CS is currently controversial, because there are few large-sample cohort studies on the general population that clarify this association. The prevalence of pain 


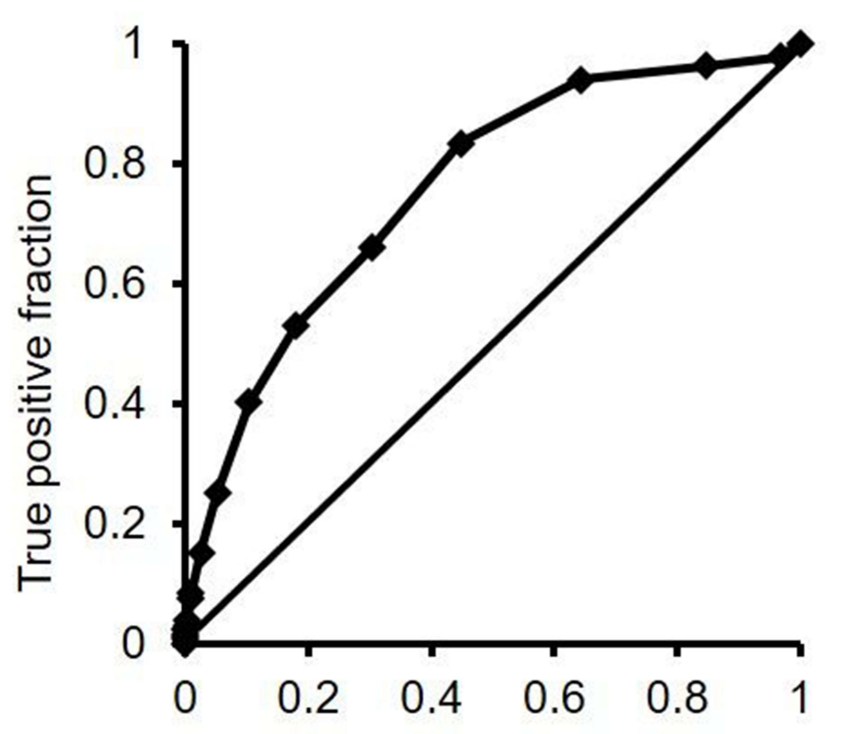

False positive fraction

Figure 3 The receiver operating characteristic (ROC) curve of the PSQI score for detecting the presence of central sensitization (CS). To estimate the cut-off value of the PSQI score for determining the presence of CS, ROC analysis was performed with the PSQI total score as the testing variable and the presence of CS as the diseased condition. The area under the curve was 0.750 ( $95 \%$ confidence interval: $0.713-0.801, p<0.001$ ). The cut-off point was defined as the nearest point to the true positive, estimated as 5 points with an odds ratio of 4.43 .

sensitization has been reported to be higher in terminal OA patients than in asymptomatic controls, ${ }^{41}$ and it was shown to be associated with radiographic OA severity. ${ }^{41}$ In contrast, the CHECK study with 2126 subjects revealed that the pressure pain threshold and temporal summation were associated with OA-related pain, but not with radiographic OA. ${ }^{43}$ In addition, a reduced $\mathrm{CS}$ or a decrease in the pain threshold level was indicated by diminished intensity and not by radiographic severity. ${ }^{44}$ Moreover, a weak association has been propounded between the degree of joint damage and the clinical pain intensity; however, sensitization has performed a significant role in the experience of chronic joint pain by OA patients. ${ }^{5}$ In the present study, hyperalgesia around the knee joint seems to be the reason for the increased prevalence of nocturnal knee pain via sensitization in participants with CS.

A direct association between $\mathrm{CS}$ and radiographic knee OA severity was unproven in the present study. The descending pain inhibitory pathway was regarded as a potential cause for the chronic pain in knee $\mathrm{OA} ;^{45}$ additionally, $28 \%$ of the terminal arthritis patients displayed CS with a diminished QOL. ${ }^{20}$ The association between knee OA severity and the comorbidity of CS is presently controversial. In patients, the degree of sensitization was noted to correlate with the clinical pain reports, but not with the radiographic findings. ${ }^{46}$ In addition, increased sensitivity was observed to occur during elevated pain and low-grade knee OA; this suggests the apparent role of $\mathrm{CS}$ in elevated pain, regardless of the evidence of pathological OA changes. ${ }^{44}$ Previous studies support our results that the prevalence of CS did not increase with the KL grade in the general population; however, the nocturnal knee symptoms escalated through pain sensitization. In contrast, the prevalence of nocturnal knee pain and disability with respect to knee OA severity was previously demonstrated. ${ }^{10}$ The finding that the prevalence of CS is weakly dependent on age in men, but not the severity of radiographic knee OA, would be considered as influenced by the other musculoskeletal or psychiatry diseases.

In the present study, the cut-off value of the PSQI score for detecting the presence of CS was 4 points. Typically, this is set at 6 for sleep disturbance; therefore, this study finding is worthy of further evaluation. For the purpose of knee surgery, it is critical to evaluate the sleep quality and nocturnal symptoms. Based on previous clinical studies, the presence of preoperative CS would remain a potential risk for chronic post-surgical pain following total knee arthroplasty. ${ }^{19,33,34}$ Preoperative evaluations and interventions for CS or nocturnal symptoms are necessary for satisfactory clinical outcomes, regardless of the radiographic knee OA severity. Preoperative administration of duloxetine was reported to reduce the risk of chronic post-surgical pain. ${ }^{47}$ The present study demonstrated CS-diminished sleep quality, as evaluated by PSQI; this may be an indicator for CS.

The present study contains several limitations. First, the study sample comprised the Japanese general population; data regarding the patients' background characteristics and confounding factors were unavailable. Second, the evaluation of CS was conducted exclusively by selfadministered questionnaires. The power of the CSI-9 is likely to be relatively weak in comparison with available physical examinations for pain stimulation and evaluation, such as temporal summation, pressure pain thresholds, quantitative sensory testing, mild pain detection, and pressure tolerance thresholds, and the visual analog scale. $^{5,40,42}$ However, performing these is difficult in a large-sample cohort study such as ours. Third, the psychological condition of the patients was required to be examined as some $\mathrm{OA}$ patients may display 
a catastrophizing or depressive background for chronic pain. ${ }^{21}$ Fourth, the imaging examination was limited to radiography; however, magnetic resonance imaging or ultrasound sonography is advantageous in detecting minute structural changes. Therefore, detailed etiology related to CS or nocturnal knee symptoms may be underestimated. Fifth, owing to the small number of patients with a KL grade 4, a positive ratio of CSI-9 was not considered for the statistical accuracy, and hence KL grade was summarized as the knee OA severity. This limitation is attributed to the epidemiological nature of the study targeting the general population whereas a clinical study would feature confirmed patients. Finally, due to the cross-sectional nature of the study, we could not identify the causal relationship between CS and nocturnal knee symptoms in participants with OA; future longitudinal studies are required.

\section{Conclusion}

Radiographic knee OA severity was correlated with an increase in nocturnal knee pain and disability; however, its association with the prevalence of CS was unproven. The combination of knee OA and CS enhanced the occurrence of nocturnal knee symptoms and diminished sleep quality. A PSQI score $>4$ points could detect a significant risk of potential CS and hence be an effective screening tool.

\section{Abbreviations}

CS, central sensitization; CSI, central sensitization inventory A; ICC, interclass correlation coefficient; KL, KellgrenLawrence; KOOS, knee injury and osteoarthritis outcome score; OA, osteoarthritis; PSQI, Pittsburgh sleep quality index; QOL, quality of life; SMI, skeletal muscle index.

\section{Data Sharing Statement}

The study protocol, statistical analysis, and data supporting the findings of this study are available from the corresponding author upon reasonable request.

\section{Ethics Approval and Informed Consent}

The study was performed in agreement with the 1964 Helsinki Declaration and later amendments or comparable ethical standards. Approval was obtained from the ethics committee of the Hirosaki University Graduate School of Medicine. Written informed consent was obtained from all participants.

\section{Acknowledgments}

The authors thank the medical staff who supported the conduct of the Iwaki Health Promotion Project, as well as Editage for English language editing.

\section{Author Contributions}

All authors made a significant contribution to the work reported, whether that is in the conception, study design, execution, acquisition of data, analysis and interpretation, or in all these areas; took part in drafting, revising or critically reviewing the article; gave final approval of the version to be published; have agreed on the journal to which the article has been submitted; and agree to be accountable for all aspects of the work.

\section{Funding}

The present study was supported in part by JST COI Grant Number JPMJCE1302, a Grant-in-Aid from the Japanese Society for the Promotion of Science (Nos. 21500676, 18K16606, 18K09091), the Health Labor Sciences Research Grant, a JOA-Subsidized Science Project Research from the Japanese Orthopedic Association (2018-4), and the Grant of Japan Orthopedics and Traumatology Research Foundation (No. 421).

\section{Disclosure}

The authors declare that they have no competing interests.

\section{References}

1. Lawrence RC, Helmick CG, Arnett FC, et al. Estimates of the prevalence of arthritis and selected musculoskeletal disorders in the United States. Arthritis Rheum. 1998;41(5):778-799. doi:10.1002/ 1529-0131(199805)41:5<778::AID-ART4>3.0.CO;2-V

2. Yoshimura N, Muraki S, Oka H, et al. Prevalence of knee osteoarthritis, lumbar spondylosis, and osteoporosis in Japanese men and women: the research on osteoarthritis/osteoporosis against disability study. $J$ Bone Miner Metab. 2009;27(5):620-628. doi:10.1007/s00774-0090080-8

3. Hiligsmann M, Cooper C, Arden N, et al. Health economics in the field of osteoarthritis: an expert's consensus paper from the European Society for Clinical and Economic Aspects of Osteoporosis and Osteoarthritis (ESCEO). Semin Arthritis Rheum. 2013;43 (3):303-313. doi:10.1016/j.semarthrit.2013.07.003

4. Neogi T. The epidemiology and impact of pain in osteoarthritis. Osteoarthritis Cartilage. 2013;21(9):1145-1153. doi:10.1016/j. joca.2013.03.018

5. Arendt-Nielsen L, Skou ST, Nielsen TA, Petersen KK. Altered central sensitization and pain modulation in the CNS in chronic joint pain. Curr Osteoporos Rep. 2015;13(4):225-234. doi:10.1007/s11914-0150276-x

6. Muraki S, Oka H, Akune T, et al. Prevalence of radiographic knee osteoarthritis and its association with knee pain in the elderly of Japanese population-based cohorts: the ROAD study. Osteoarthritis Cartilage. 2009;17(9):1137-1143. doi:10.1016/j.joca.2009.04.005 
7. Jinks C, Jordan K, Croft P. Osteoarthritis as a public health problem: the impact of developing knee pain on physical function in adults living in the community: (KNEST 3). Rheumatology. 2007;46 (5):877-881. doi:10.1093/rheumatology/kem013

8. Sharma L, Cahue S, Song J, Hayes K, Pai YC, Dunlop D. Physical functioning over three years in knee osteoarthritis: role of psychosocial, local mechanical, and neuromuscular factors. Arthritis Rheum. 2003;48(12):3359-3370. doi:10.1002/art.11420

9. Sasaki E, Ishibashi Y, Tsuda E, et al. Evaluation of locomotive disability using loco-check: a cross-sectional study in the Japanese general population. J Orthop Sci. 2013;18(1):121-129. doi:10.1007/ s00776-012-0329-2

10. Sasaki E, Tsuda E, Yamamoto Y, et al. Nocturnal knee pain increases with the severity of knee osteoarthritis, disturbing patient sleep quality. Arthritis Care Res. 2014;66(7):1027-1032. doi:10.1002/ acr. 22258

11. Hawker GA, Stewart L, French MR, et al. Understanding the pain experience in hip and knee osteoarthritis-an OARSI/OMERACT initiative. Osteoarthritis Cartilage. 2008;16(4):415-422. doi:10.1016/j. joca.2007.12.017

12. Woolhead G, Gooberman-Hill R, Dieppe P, Hawker G. Night pain in hip and knee osteoarthritis: a focus group study. Arthritis Care Res. 2010;62(7):944-949. doi:10.1002/acr.20164

13. Power JD, Perruccio AV, Badley EM. Pain as a mediator of sleep problems in arthritis and other chronic conditions. Arthritis Rheum. 2005;53(6):911-919. doi:10.1002/art.21584

14. Bajaj P, Bajaj P, Graven-Nielsen T, Arendt-Nielsen L. Osteoarthritis and its association with muscle hyperalgesia: an experimental controlled study. Pain. 2001;93(2):107-114. doi:10.1016/S0304 3959(01)00300-1

15. Imamura M, Imamura ST, Kaziyama HH, et al. Impact of nervous system hyperalgesia on pain, disability, and quality of life in patients with knee osteoarthritis: a controlled analysis. Arthritis Rheum. 2008;59(10):1424-1431. doi:10.1002/art.24120

16. International Association for the Study of Pain. IASP Terminology. Available from: https://www.iasp-pain.org/Education/Content.aspx? ItemNumber=1698\#Sensitization. Accessed August 10, 2021.

17. Latremoliere A, Woolf CJ. Central sensitization: a generator of pain hypersensitivity by central neural plasticity. $J$ Pain. 2009;10 (9):895-926. doi:10.1016/j.jpain.2009.06.012

18. Loeser JD, Treede RD. The kyoto protocol of IASP basic pain terminology. Pain. 2008;137(3):473-477. doi:10.1016/j. pain.2008.04.025

19. Petersen KK, Arendt-Nielsen L, Simonsen O, Wilder-Smith O, Laursen MB. Presurgical assessment of temporal summation of pain predicts the development of chronic postoperative pain 12 months after total knee replacement. Pain. 2015;156(1):55-61. doi:10.1016/j. pain.0000000000000022

20. Hochman JR, Gagliese L, Davis AM, Hawker GA. Neuropathic pain symptoms in a community knee OA cohort. Osteoarthritis Cartilage. 2011;19(6):647-654. doi:10.1016/j.joca.2011.03.007

21. Campbell CM, Buenaver LF, Finan P, et al. Sleep, pain catastrophizing, and central sensitization in knee osteoarthritis patients with and without insomnia. Arthritis Care Res. 2015;67(10):1387-1396. doi:10.1002/acr.22609

22. Nijs J, Van Houdenhove B, Oostendorp RA. Recognition of central sensitization in patients with musculoskeletal pain: application of pain neurophysiology in manual therapy practice. Man Ther. 2010;15(2):135-141. doi:10.1016/j.math.2009.12.001

23. Sasaki E, Ota S, Chiba D, et al. Early knee osteoarthritis prevalence is highest among middle-aged adult females with obesity based on new set of diagnostic criteria from a large sample cohort study in the Japanese general population. Knee Surg Sports Traumatol Arthrosc. 2020;28(3):984-994. doi:10.1007/s00167-019-05614-z
24. Kellgren JH, Lawrence RC. Radiological assessment of osteo-arthrosis. Ann Rheum Dis. 1957;16(4):494-502. doi:10.1136/ ard.16.4.494

25. Inoue R, Ishibashi Y, Tsuda E, et al. Knee osteoarthritis, knee joint pain and aging in relation to increasing serum hyaluronan level in the Japanese population. Osteoarthritis Cartilage. 2011;19(1):51-57. doi:10.1016/j.joca.2010.10.021

26. Mayer TG, Neblett R, Cohen H, et al. The development and psychometric validation of the central sensitization inventory. Pain Pract. 2012;12(4):276-285. doi:10.1111/j.1533-2500.2011.00493.x

27. Nishigami T, Tanaka K, Mibu A, Manfuku M, Yono S, Tanabe A. Development and psychometric properties of short form of central sensitization inventory in participants with musculoskeletal pain: a cross-sectional study. PLoS One. 2018;13(7):e0200152. doi:10.1371/ journal.pone.0200152

28. Nakamura N, Takeuchi R, Sawaguchi T, Ishikawa H, Saito T, Goldhahn S. Cross-cultural adaptation and validation of the Japanese Knee Injury and Osteoarthritis Outcome Score (KOOS). J Orthop Sci. 2011;16(5):516-523. doi:10.1007/s00776-011-0112-9

29. Roos EM, Roos HP, Lohmander LS, Ekdahl C, Beynnon BD. Knee Injury and Osteoarthritis Outcome Score (KOOS)-development of a self-administered outcome measure. J Orthop Sports Phys Ther. 1998;28(2):88-96. doi:10.2519/jospt.1998.28.2.88

30. Omachi TA. Measures of sleep in rheumatologic diseases: Epworth Sleepiness Scale (ESS), Functional Outcome of Sleep Questionnaire (FOSQ), Insomnia Severity Index (ISI), and Pittsburgh Sleep Quality Index (PSQI). Arthritis Care Res. 2011;63 Suppl 11(11):S287-96. doi:10.1002/acr.20544

31. Doi Y, Minowa M, Uchiyama M, et al. Psychometric assessment of subjective sleep quality using the Japanese version of the Pittsburgh Sleep Quality Index (PSQI-J) in psychiatric disordered and control subjects. Psychiatry Res. 2000;97(2-3):165-172. doi:10.1016/s01651781(00)00232-8

32. Buysse DJ, Reynolds CF 3rd, Monk TH, Berman SR, Kupfer DJ. The Pittsburgh Sleep Quality Index: a new instrument for psychiatric practice and research. Psychiatry Res. 1989;28(2):193-213. doi:10.1016/0165-1781(89)90047-4.

33. Kim SH, Yoon KB, Yoon DM, Yoo JH, Ahn KR. Influence of centrally mediated symptoms on postoperative pain in osteoarthritis patients undergoing total knee arthroplasty: a prospective observational evaluation. Pain Pract. 2015;15(6):E46-E53. doi:10.1111/papr.12311

34. Werner MU, Kongsgaard UEI. Defining persistent post-surgical pain: is an update required? Br J Anaesth. 2014;113(1):1-4. doi:10.1093/ bja/aeu012

35. Wright JG, Coyte P, Hawker G, et al. Variation in orthopedic surgeons' perceptions of the indications for and outcomes of knee replacement. CMAJ. 1995;152(5):687-697.

36. Mancuso CA, Ranawat CS, Esdaile JM, Johanson NA, Charlson ME. Indications for total hip and total knee arthroplasties. Results of orthopaedic surveys. J Arthroplasty. 1996;11(1):34-46. doi:10.1016/ s0883-5403(96)80159-8

37. Gillingham SJ, Alvi F, Lovell ME. The effect of increasing age on nocturnal joint pain in patients about to undergo hip or knee joint arthroplasty. Arch Gerontol Geriatr. 2010;50(1):34-35. doi:10.1016/j. archger.2009.01.006

38. Enomoto H, Fujikoshi S, Tsuji T, Sasaki N, Tokuoka H, Uchio Y. Efficacy of duloxetine by prior NSAID use in the treatment of chronic osteoarthritis knee pain: a post hoc subgroup analysis of a randomized, placebo-controlled, Phase 3 study in Japan. J Orthop Sci. 2018;23(6):1019-1026. doi:10.1016/j.jos.2018.07.008

39. Uchio Y, Enomoto H, Alev L, et al. A randomized, double-blind, placebo-controlled Phase III trial of duloxetine in Japanese patients with knee pain due to osteoarthritis. J Pain Res. 2018;11:809-821. doi:10.2147/JPR.S164128 
40. Arendt-Nielsen L. Pain sensitisation in osteoarthritis. Clin Exp Rheumatol. 2017;35 Suppl 107(5):68-74.

41. Petersen KK, Arendt-Nielsen L, Finocchietti S, et al. Age interactions on pain sensitization in patients with severe knee osteoarthritis and controls. Clin J Pain. 2017;33(12):1081-1087. doi:10.1097/ AJP.0000000000000495

42. Fingleton C, Smart K, Moloney N, Fullen BM, Doody C. Pain sensitization in people with knee osteoarthritis: a systematic review and meta-analysis. Osteoarthritis Cartilage. 2015;23(7):1043-1056. doi:10.1016/j.joca.2015.02.163

43. Neogi T, Frey-Law L, Scholz J, et al.; Multicenter Osteoarthritis (MOST) Study. Sensitivity and sensitisation in relation to pain severity in knee osteoarthritis: trait or state? Ann Rheum Dis. 2015;74 (4):682-688. doi:10.1136/annrheumdis-2013-204191.
44. Finan PH, Buenaver LF, Bounds SC, et al. Discordance between pain and radiographic severity in knee osteoarthritis: findings from quantitative sensory testing of central sensitization. Arthritis Rheum. 2013;65(2):363-372. doi:10.1002/art.34646

45. Nelson FR. A background for the management of osteoarthritic knee pain. Pain Manag. 2014;4(6):427-436. doi:10.2217/pmt.14.40

46. Woolf CJ. Central sensitization: implications for the diagnosis and treatment of pain. Pain. 2011;152(3 Suppl):S2-S15. doi:10.1016/j. pain.2010.09.030

47. Koh IJ, Kim MS, Sohn S, Song KY, Choi NY, In Y. Duloxetine reduces pain and improves quality of recovery following total knee arthroplasty in centrally sensitized patients: a prospective, randomized controlled study. J Bone Joint Surg Am. 2019;101(1):64-73. doi:10.2106/JBJS.18.00347

\section{Publish your work in this journal}

The Journal of Pain Research is an international, peer reviewed, open access, online journal that welcomes laboratory and clinical findings in the fields of pain research and the prevention and management of pain. Original research, reviews, symposium reports, hypothesis formation and commentaries are all considered for publication. The manuscript management system is completely online and includes a very quick and fair peer-review system, which is all easy to use. Visit http:// www.dovepress.com/testimonials.php to read real quotes from published authors. 